\title{
Clinical and radiological features of orbito-temporal neurofibromatosis
}

\author{
Isha Chaturvedi ${ }^{1}$, Kumudini Sharma ${ }^{2}$ \\ From ${ }^{1}$ Senior Resident, ${ }^{2}$ Professor and Head, Department of Ophthalmology, Sanjay Gandhi Postgraduate Institute of Medical Sciences, Lucknow, \\ Uttar Pradesh, India
}

\begin{abstract}
Neurofibromatosis- 1 (NF-1) is an autosomal dominant condition belonging to the well-known group of neurocutaneous syndromes. It affects 1 in 3500 persons. It is caused by a mutation in the neurofibromin gene localized to chromosome 17q11.2. which codes for a protein involved in the normal cell cycle regulation. Therefore NF-1 gene mutations predispose the individual to various benign and malignant tumors. The diagnosis of NF-1 remains largely clinical, given its characteristic lesions. Here we describe one such case with hallmark lesions of NF-1. A 12-year-old boy presented with a slow-growing and progressive right upper lid mass causing severe drooping, vision impairment and facial disfiguration. On detailed ophthalmic examination he was found to have severe ptosis, Lisch nodules and keratoconus. A systemic examination revealed café au lait spots and axillary freckling. Radiological imaging showed sphenoid wing dysplasia, encephalocele and optic pathway glioma. The patient was taken up for debulking surgery and a referral to the neurosurgery department was sent. He has been on follow up for last 12 months. This case first presented to an ophthalmology clinic and thus, as an ophthalmologist, keeping in mind the associated intraorbital and intracranial pathology can be life-saving. A multidisciplinary approach is needed in the management of such severe cases of NF-1.
\end{abstract}

Keywords: Optic pathway glioma (OPG), Plexiform neurofibroma, Proptosis, Sphenoid wing dysplasia

$\mathrm{N}$ eurofibromatosis-1 (NF-1) is a relatively common multisystem disease affecting 1 in 3500 persons [1]. It was first described and coined by the German pathologist, Von Recklinghausen [2] in 1882, who first understood the origin of skin tumors and named them neurofibromas. However, the history of NF-1 can be traced to ancient times, if non-scientific descriptions of grotesque persons or erroneously diagnosed cases such as that of Joseph Merrick ("elephant man") are considered [3]. The diagnostic criteria currently used for diagnosis were presented by the National Institute of Health (NIH) in 1987 [4].

An individual with at least two of the following criteria is considered affected by the disease: At least six café-au-lait spots with a minimum diameter of $0.5 \mathrm{~cm}$ before puberty or minimum diameter of $1.5 \mathrm{~cm}$ following puberty, inguinal and/or axillary freckling, at least two Lisch nodules, optic pathway glioma (OPG), and typical bony lesions such as sphenoid wing dysplasia, pseudoarthritis of the tibia, and a first-degree relative with NF1. Severe cases of NF-1 often present to the ophthalmologist with facial disfigurement. It is imperative at the time, to consider any underlying intracranial pathology and has a multidisciplinary approach to manage and rehabilitate such patients. Here, we report a case of NF-1 who

\section{Access this article online}

Received - 05 May 2021

Initial Review - 20 May 2021

Accepted - 27 May 2021

DOI: 10.32677/IJCR.2021.v07.i06.016 presented with a massive plexiform neurofibroma $(\mathrm{PN})$ of the right upper eyelid and a proptosed eyeball.

A 12-year-old boy presented to the ophthalmology clinic with a gradually progressive mass involving the right eyelid, over the past 5 years. On examination, it was a boggy swelling on the right upper lid causing severe ptosis and downward displacement of the globe (Fig. 1). The swelling involved the right side of the forehead, brow, and upper lid, measuring approximately

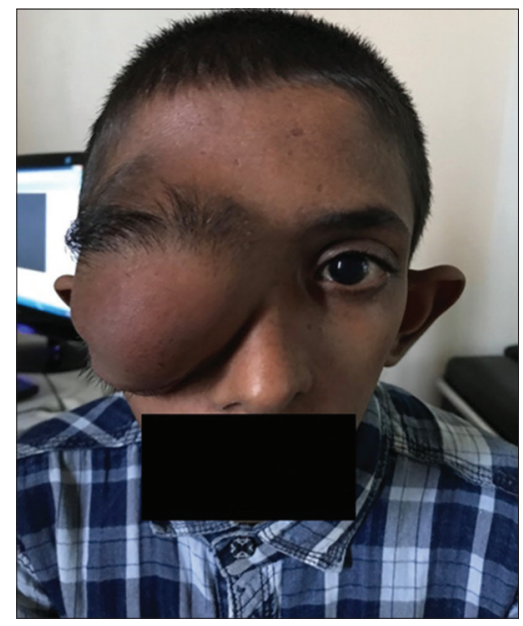

Figure 1: Plexiform neurofibroma of the right upper lid with disfiguring ptosis and eccentric proptosis

Correspondence to: Dr. Isha Chaturvedi, 17, Vishnulok Colony, Kanpur Road, Lucknow - 226 012, Uttar Pradesh, India. E-mail: ishachats.90@gmail.com

(C) 2021 Creative Commons Attribution-NonCommercial 4.0 International License (CC BY-NC-ND 4.0). 


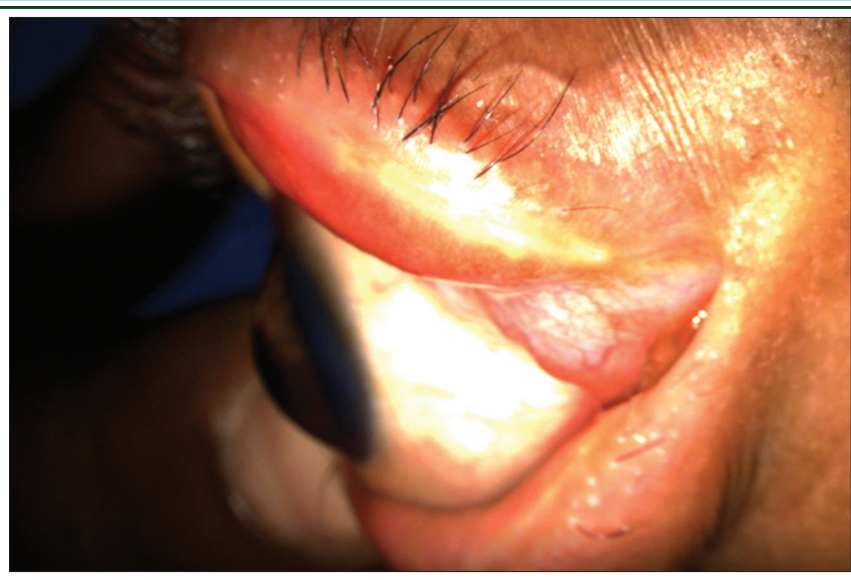

Video 1: Pulsatile proptosis caused by the encephalocele herniating into right orbit

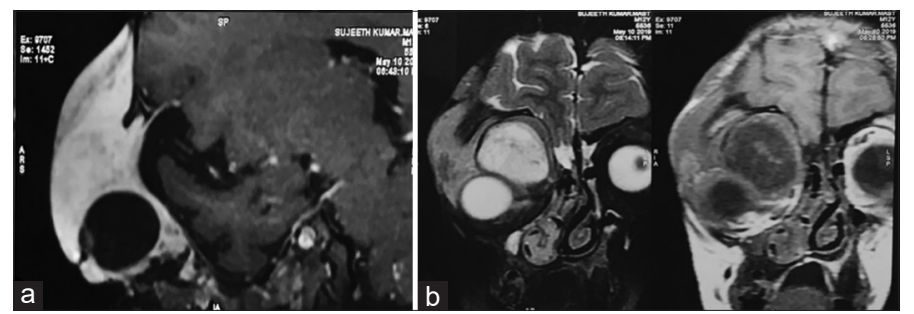

Figure 2: (a) Absent greater wing of sphenoid with temporal lobe herniation into the orbit; (b) a homogenous right preseptal and periocular lesion showing isointense signal in $\mathrm{T} 1$ (intermediate signal in T2-weighted image). It shows homogenous contrast enhancement

$12-13 \mathrm{~cm}$ vertically and $7-8 \mathrm{~cm}$ across. The mass was reducible and pulsatile (Video 1). On detailed slit lamp examination, Lisch nodules in both eyes and keratoconus in the right eye were observed. Systemic examination revealed café-au-lait spots and axillary freckling. His best-corrected visual acuity in the right and left eye was $6 / 60$ and $6 / 6$, respectively. Fundus examination was normal.

Considering his age, we thought the cause of decreased vision in the right eye to be stimulus deprivation amblyopia due to severe ptosis. Nevertheless, keeping in mind, the occurrence of sphenoid dysplasia, OPG, and the risk of malignant transformation in such patients, a magnetic resonance imaging brain and orbit (plain and contrast) was done. Imaging revealed an absent greater wing of sphenoid with temporal lobe herniation into the orbit causing proptosis (Fig. 2a). A homogenous right preseptal and periocular lesion was noted which showed contrast enhancement (Fig. 2b).
The patient underwent tumor debulking and reconstructive surgery in consultation with a neurosurgeon. Since then, he has been on follow-up.

The frequency of café-au-lait spots (95-100\%), axillary freckling (81\%), Lisch nodules (50-90\%), osseous lesion (60\%), and optic pathway gliomas (15\%) in NF-1 has been documented in earlier studies [5]. The patient was diagnosed as a case of orbito-temporal NF in accordance with the NIH criteria. PNs can progressively involve the eyelid, orbit, periorbital, and facial region causing disfigurement. They are known in the medical literature by many terms, namely, orbitotemporal PNs, orbitopalpebral NF, orbitotemporal neurofibromatosis, orbital neurofibromas, and orbital-periorbital plexiform neurofibroma [6].

\section{AUTHOR CONTRIBUTIONS}

Dr. Isha Chaturvedi - Senior Resident, Ophthalmology, Sanjay Gandhi Post Graduate Institute of Medical Sciences.

Dr. Kumudini Sharma - Professor and Head, Ophthalmology, Sanjay Gandhi Post Graduate Institute of Medical Sciences.

\section{REFERENCES}

1. Riccardi VM. Neurofibromatosis: Past, present and future. N Engl J Med 1991;324:1238-85.

2. Von Recklinghausen FD. Ber die Multiplen Fibrome der Haut und Ihre Beziehung zu den Multiplen Neuromen. Berlin: Hirschwald; 1882.

3. Martino R, Andrea DP, Rosario C, Agata P. Early history of the different forms of neurofibromatosis from ancient Egypt to the British empire and beyond: First descriptions, medical curiosities, misconceptions, landmarks, and the persons behind the syndromes. Am J Med Genet A 2018;176:515-50.

4. Neurofibromatosis. Conference statement. National institutes of health consensus development conference. Arch Neurol 1988;45:575-8.

5. Kinori M, Hodgson N, Zeid JL. Ophthalmic manifestations in neurofibromatosis Type 1. Surv Ophthalmol 2018;63:518-33.

6. Robert A, James K, Michael F, Gena H, Eva D, Roger P, et al. Orbital/ periorbital plexiform neurofibromas in children with neurofibromatosis Type 1 multidisciplinary recommendations for care. Ophthalmology 2017; 124:123-32.

Funding: None; Conflicts of Interest: None Stated.

How to cite this article: Chaturvedi I, Sharma K. Clinical and radiological features of orbito-temporal neurofibromatosis. Indian $\mathrm{J}$ Case Reports. 2021;7(6):268-269. 